Published in final edited form as:

Nutrition. 2020 October ; 78: 110819. doi:10.1016/j.nut.2020.110819.

\title{
Development of the Dietitian Integrated Evaluation Tool for Gluten-Free Diets (DIET-GFD)
}

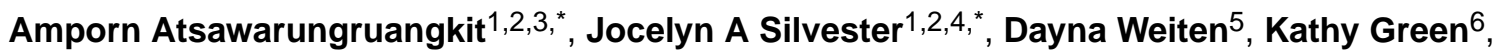 \\ Kaitlyn Wilkey ${ }^{1}$, Lisa Rigaux ${ }^{6}$, Charles N Bernstein ${ }^{7}$, Lesley A Graff ${ }^{8}$, John R Walker ${ }^{8}$, \\ Donald R Duerksen ${ }^{6,7}$ \\ ${ }^{1}$ Harvard Medical School Celiac Research Program, Boston, Massachusetts, USA \\ ${ }^{2}$ Beth Israel Deaconess Medical Center, Boston, Massachusetts, USA \\ ${ }^{3}$ MetroWest Medical Center, Framingham, Massachusetts, USA \\ ${ }^{4}$ Boston Children's Hospital, Boston, Massachusetts, USA \\ ${ }^{5}$ Grace General Hospital, Winnipeg, Manitoba, Canada \\ ${ }^{6}$ St Boniface Hospital, Winnipeg, Manitoba, Canada \\ ${ }^{7}$ Department of Internal Medicine, Max Rady College of Medicine, Rady Faculty of Health \\ Sciences, University of Manitoba, Winnipeg, Manitoba, Canada \\ ${ }^{8}$ Department of Clinical Health Psychology, Max Rady College of Medicine, Rady Faculty of \\ Health Sciences, University of Manitoba, Winnipeg, Manitoba, Canada
}

\begin{abstract}
Objective-Celiac disease (CD) treatment involves a gluten-free diet (GFD). There is no standardized tool for dietitians to objectively grade GFD adherence. This study aimed to develop a standardized tool for dietitians to evaluate and communicate GFD adherence.

Research Methods \& Procedures-Participants were recruited from the Manitoba Celiac Disease Cohort. Using a consensus process, an expert panel of gastroenterologists, dietitians, clinical health psychologists and persons with CD developed the DIET-GFD. Two dietitians then
\end{abstract}

\footnotetext{
"Corresponding Author: Jocelyn A. Silvester MD PhD, Assistant Professor of Pediatrics, Boston Children's Hospital, Harvard Medical School, 300 Longwood Avenue, Boston MA, 02115, 617-355-6058, jocelyn.silvester@ childrens.harvard.edu. CRediT Author Statement

Amporn Atsawarungruangkit: Methodology, data curation, writing - original draft, writing - review and editing, visualization. Charles Bernstein: Formal analysis, writing — review and editing

Donald Duerksen: Conceptualization, methodology, formal analysis, data curation, writing — review and editing, supervision Kathy Green: Conceptualization, methodology, data curation, writing — review and editing

Lesley Graff: Conceptualization, methodology, formal analysis, writing—review and editing

Lisa Rigaux: Conceptualization, investigation, data curation, writing-review and editing

Jocelyn Silvester: Conceptualization, methodology, formal analysis, data curation, writing — review and editing, supervision

John Walker: Conceptualization, methodology, writing - review and editing

Dayna Weiten: Conceptualization, methodology, formal analysis, data curation, writing—review and editing

Kaitlyn Wilkey: Methodology, data curation, writing - review and editing

* These authors contributed equally to this work

Publisher's Disclaimer: This is a PDF file of an unedited manuscript that has been accepted for publication. As a service to our customers we are providing this early version of the manuscript. The manuscript will undergo copyediting, typesetting, and review of the resulting proof before it is published in its final form. Please note that during the production process errors may be discovered which could affect the content, and all legal disclaimers that apply to the journal pertain.
} 
performed duplicate assessments of 27 newly diagnosed participants who had been advised to follow a GFD. The global adherence scale was further revised following panel discussions of the cases where there was uncertainty or discordance on dietitian ratings. The scoring system was then evaluated using duplicate assessments of an additional $37 \mathrm{CD}$ participants. Inter-rater agreement was assessed using square-weight Cohen's kappa.

Results-The DIET-GFD includes features related to frequency and quantity of gluten ingestion based upon self-report and food frequency evaluation, shopping and dining habits, how and where food is prepared and consumed, eating behaviors, and label reading skills. The DIET-GFD global assessment is reported using a 10-point ordinal descriptive scale ranging from 1 (takes few precautions and regularly eats gluten) to 10 (no gluten in kitchen and rarely eats food prepared outside the home). The kappa of DIET-GFD global assessment was 0.845 indicating excellent agreement.

Conclusions-DIET-GFD is a useful tool for dietitians to evaluate GFD adherence. Further studies are needed to confirm that the score from DIET-GFD is reliable across various settings.

\section{Keywords}

Gluten-free diet; celiac disease; adherence assessment; interobserver agreement

\section{Introduction}

Celiac disease (CD) is a chronic immune disease characterized by gluten-induced small intestinal villous atrophy. Strict gluten avoidance is associated with clinical improvement, mucosal recovery,[1,2] diminished long term complications, [3-7] including malignancy, $[3,5,8]$ and improved quality of life.[9-12] Thus, dietary intervention through lifelong adherence to a strict gluten-free diet (GFD) is the cornerstone of long-term management of patients with CD. Despite reported good adherence to GFD for a period of two years, there are some CD patients who do not respond, as demonstrated by persistent symptoms, abnormal serologic tests, and histologic abnormalities. [4,13-17]. Moreover, multiple studies have shown that a large proportion of CD patients may stop adhering to GFD at some point in their lifetime. $[15,18,19]$ Following a GFD is practically challenging and requires knowledge not only of food ingredients, but also of food preparation.

In clinical practice, available methods for monitoring GFD adherence include patient selfreport, consultation with a dietitian, a combination of history and CD serology (IgA tissue trans glutaminase (tTG) or IgA (or IgG) deamidated gliadin peptide (DGP) antibodies), and biopsy evaluation.[13] Tests to measure gluten excretion in urine and stool are in development, and not yet widely available.[20,21] Unfortunately, patient self-report tends to overestimate GFD adherence since it cannot capture unintentional gluten ingestion.[22] CD serology tests have low sensitivity and specificity for detecting ongoing gluten exposure.[23] Intestinal biopsy is invasive, costly and not without risk, thus the role of follow-up intestinal biopsy in monitoring patients with $\mathrm{CD}$ is controversial.[13,24]

Currently, consultation with a skilled dietitian is considered the clinical gold standard for monitoring adherence to GFD.[13,25] With limited numbers of dietitians who specialize in 
$\mathrm{CD}$, it is very challenging for patients to get the proper guidance on lifelong adherence to GFD. There is no consensus on the skills required, depth of knowledge, or methodology for a dietitian to assess GFD adherence.[15] Even in research settings, methodology is poorly described and varies widely.[24]

Lack of standardization and lack of consensus regarding items that should be included to properly evaluate GFD adherence severely limits the accuracy, reproducibility and comparability of GFD adherence assessments in different settings administered by different healthcare providers. Strikingly, interobserver correlation of a dietitian assessment of GFD adherence has not been reported. With the growing number of patients diagnosed with $C D$, and the need for ongoing lifetime gluten avoidance to manage the disease, there is an increasing need for a reliable diet assessment tool. The existence of standardized tools that allow different dietitians to efficiently assess GFD adherence with high inter-rater agreement would be beneficial to both healthcare providers and patients. The aim of this study was to evaluate the interobserver agreement of a newly developed integrated GFD evaluation tool.

\section{Materials and Methods}

\section{Tool development}

An expert panel composed of gastroenterologists, dietitians with expertise in monitoring patients on GFDs, clinical health psychologists and persons with CD was convened to identify elements integral to assessment of GFD adherence. A literature review was performed and the following domains relevant to GFD adherence were identified: (1) Grocery shopping and label reading skills; (2) Managing gluten in the home and kitchen; (3) Eating outside the home; (4) Ability to ascertain if food prepared by others is gluten-free; (5) Awareness and prevention of cross-contact; and (6) Intentional dietary lapses. The expert panel defined a 10-point ordinal descriptive scale for dietary adherence. Textual revisions were made following review by members of the Canadian Celiac Association.

\section{Piloting and scale refinement}

This study was conducted in the context of the Manitoba Celiac Disease Cohort prospective observational inception cohort study of newly diagnosed patients with CD who have been advised to follow a gluten-free diet. The Manitoba Celiac Disease Cohort includes individuals with a biopsy-confirmed celiac disease diagnosis (Marsh III histology) and elevated tTG and/or endomysial antibodies. Persons unable to complete written surveys or oral interviews in English, unable to attend follow-up appointments, or who had been trying to follow a gluten-free diet for greater than six weeks prior to study entry were excluded. The study protocol involves evaluations 6, 12 and 24 months after diagnosis, necessitating a standardized dietitian assessment to compare outcomes over time. Items from the diet assessment were integrated into the interview, self-report survey, 3-day food record, and grocery label quiz which participants completed for this study. Two dietitians (KG and DW) with specific expertise and experience monitoring patients on GFDs each independently classified the adherence of 15 cohort study participants with widely varying gluten intake, using the 10-point ordinal descriptive scale developed (see Table 1). Participants for this phase were identified based upon dietitian assessed adherence (a single dietitian) to ensure 
that those who were less adherent to a GFD were included. The expert panel then reconvened to discuss challenges and resolve differences in scoring. The scoring system was then validated using independent duplicate assessments of a different subset of cohort participants with biopsy-confirmed CD who had been advised to follow a GFD. Participants for this phase were a non-overlapping random 20\% sample of the Manitoba Celiac Disease Cohort with study visits after those included in the derivation cohort.

More specifically, using data from the 3-day food record and standardized food frequency questionnaire (FFQ), each dietitian assessor independently estimated the quantity of gluten consumed by each participant during the previous 3 months, using a categorical rating system that semi-quantified gluten intake based on bread equivalency [no gluten; crumbs (up to $50 \mathrm{mg}$ ); less than $1 / 4$ slice of bread $(500 \mathrm{mg})$; less than $1 / 2$ slice of bread $(1000 \mathrm{mg})$; less than 1 slice of bread ( 2 grams); less than 5 slices of bread ( 10 grams); less than 10 slices of bread (20 grams), greater than 10 slices of bread]. In addition, given that one of the purposes of GFD assessment is to identify behaviors which place a patient at risk of gluten consumption, the dietitian assessors also categorized the risk of gluten exposure in each of 4 sub-domains (home/kitchen, other peoples' homes, restaurants, shopping/label reading) as none, low, medium or high.

Shopping/label reading assessment was based upon self-reported behavior as well as performance in correctly identifying whether items in a basket of 25 goods from a local grocery store contained gluten. Participants were presented with a basket of 25 groceries procured locally and asked to determine whether they were gluten-free based upon product packaging and labeling. For products which were thought not to be gluten-free, participants indicated which ingredients they thought contained gluten. To reduce learning effects, different products were used at each study visit. Dietitians with specific training in glutenfree diets determined which ingredients contained gluten based upon current Canadian regulations for determining whether a product can be labelled gluten-free. Scoring was based upon yes/no responses; therefore, a patient would be scored as having identified a gluten-containing food item correctly, even if the details were incorrect (e.g., specific ingredient they thought contained gluten was in fact gluten-free, but item had a different gluten-containing ingredient).

Participants were also asked to rate their own GFD adherence using the Gluten-Free Eating Assessment Tool (GF-EAT)[26], a 10-point ordinal scale similar to that used by the dietitian assessors. Additional comparators included: (1) Celiac Diet Adherence Test (CDAT),[15] a validated seven-item self-report measure with questions about symptoms and personal traits; (2) Celiac Symptom Index (CSI),[6] which includes 11 items related to specific symptoms and 5 items related to general health; (3) Modified Biagi score, a 4-point ordinal scale for evaluating GFD adherence; the last item was removed from the original Biagi questionnaire[27] because certified gluten-free food is not widely available in Canada; and, (4) serum tissue transglutaminase IgA antibody level (tTG) at the time of the dietitian assessment. All tTG assays were performed in the Immunology Laboratory at St Boniface Hospital, Winnipeg, Canada. In May 2015, the assay was changed from Immulisa (Immco Diagnostics Inc, Buffalo NY) to Bioplex 2200 (Bio-rad Laboratories (Canada) Inc, Montreal 
QC). All results are reported as multiples of the upper limit of normal (ULN) for the assay used (Immulisa 20, Bioplex 15).

\section{Statistical Analysis}

Descriptive statistics are presented as number (percentage) and median with interquartile range. Additionally, Pearson's correlation was used to illustrate the relationship among variables, including self-assessment scores (before and after visits), global ten point score assessed by two dietitians, CSI,[6] CDAT,[15] and tTG IgA ULN. Inter-rater agreement between the two dietitians was assessed using square-weight Cohen's kappa. All statistical tests were two-sided and a $p$-value of less than 0.05 was considered statistically significant. All statistical analyses were conducted using STATA version 14 (StataCorp LP, College Station, TX, USA).

\section{Ethics}

The study protocol has been approved by the University of Manitoba Health Research Ethics Board. Raw data are available from the corresponding author upon request.

\section{Results}

There were 37 unique participants who had 38 visits as part of the study (17 at 6 months; 10 at 12 months; 11 at 24 months; Table 2). One participant had both a 6-month and a 12-month study visit. Of 37 participants, $70 \%$ were female with a median age of 35 years (range 16 to 80 years). There were 5 participants identified by CSI ( 3 participants) and CDAT ( 2 participants) as having possibly inadequate GFD adherence, while there was only 1 participant with poor gluten adherence (rated lower than 5 points) on global assessment of adherence. Except for one participant who was rated 1 by both dietitians, the tTG IgA ULN at the time of visit of all other participants decreased from baseline. Moreover, the number of participants that had normal tTG IgA improved from $1(3 \%)$ at baseline to $26(68 \%)$ at the time of dietitian assessment.

All GFD adherence assessment scores and sub-scales are summarized in Table 3. Neither median nor quartiles of self-assessment of GFD adherence before and after the visit were significantly different. For global assessment of adherence, the median score assessed by dietitian 1 and dietitian 2 were 8 and 7, respectively. As presented in Figure 1 and Figure 2, the patients generally had higher scores in self-assessment of GFD adherence (before visit) compared to scores assessed by dietitians. Regarding inter-rater agreements between two dietitians, the kappa of dietitian global assessment of adherence was 0.845 (see Figure 2), which can be characterized as excellent agreement.[28] As shown in Figure 3, kappa were calculated for the sub-scales of dietitian global assessment of adherence, including risk at home $(\kappa=0.864)$, risk at others' homes $(\kappa=0.904)$, risk at shopping/label reading $(\kappa=$ $0.463)$, risk at restaurants $(\kappa=0.651)$, FFQ and food record GFD adherence estimate $(\kappa=$ $0.623)$, and FFQ and food record gluten exposure estimate $(\kappa=0.715)$.

Additionally, we summarized the correlation coefficients between dietitian assessment scores and other assessments of GFD adherence (Table 4). The dietitian assessment scores were positively correlated with self-assessment scores, but negatively correlated with CDAT 
and tTG IgA ULN at 6 months. Dietitian assessment scores had weak correlations to other factors.

\section{Discussion}

In long-term follow-up of patients with $\mathrm{CD}$, consultation with a dietitian with special expertise in gluten-free diets is currently the clinical criterion standard for monitoring GFD adherence. A standardized diet assessment tool would allow for longitudinal follow-up by different practitioners and for reliable GFD assessment by dietitians with less expertise in celiac disease. The latter is particularly important given the increasing number of individuals with $\mathrm{CD}$ and the relative lack of skilled dietitians in this area. This study describes the development of the Dietitian Integrated Evaluation tool for GFDs (DIET-GFD), which includes a 10-point ordinal global descriptive score and sub-scales related to risk of gluten exposure and estimated gluten consumption. There was very good agreement for the main score and its sub-scales between two dietitians with special expertise in gluten-free diets. To our knowledge, this is the first study to develop a standardized GFD assessment tool for dietitians to report GFD adherence in adults and to validate it by evaluating the inter-rater agreement between different dietitians. The DIET-GFD may be a useful tool for both research studies and in clinical practice where patients with CD could be evaluated by different dietitians over the course of a lifetime of follow-up.

In practice and in research, self-reported adherence is the most common proxy for dietitian assessment. This method is problematic for many reasons, not least of which is that most people with CD tend to rate their own GFD adherence very highly[22]. This ceiling effect was seen in self-assessments provided by participants in the present study. Although the correlation coefficients between dietitian scores and self-assessment scores were significantly positive, the median was higher and the interquartile range was wider for the self-assessment scores than for the dietitian global scores. Notably, the self-assessment scores before and after completing a thorough assessment of dietary habits and behaviors were almost identical; however, there was a small improvement in the correlation between dietitian assessment scores and self-assessment scores after completion of the diet assessment. This behavior suggests a learning curve as patients with $\mathrm{CD}$ gain insight into their own adherence behaviors through the process of diet assessment. This supports current guidelines which recommend regular follow-up of patients on a GFD and using a skilled dietitian to monitor GFD adherence.[13,29]

The DIET-GFD was moderately correlated with the Celiac Diet Adherence Test (CDAT), a seven-item patient-completed tool that was developed using semi-structured diet assessments that included a simple food ingredient quiz and a 3-day food record or 24-hour recall and a dynamic interview conducted by a single dietitian as the gold standard. A higher CDAT score represents worse GFD adherence, thus, the DIET-GFD and CDAT were negatively correlated. Even though both tools share the common objective to monitor GFD adherence, scores were only moderately correlated. This likely reflects the difference in item composition between the two assessments as the CDAT includes items related to headaches, low energy and self-assessment ("I do not consider myself a failure") which were significant in the regression model, yet do not assess GFD adherence specifically. In contrast, the DIET- 
GFD was based upon more objective information and the global score involves descriptive characterization of dietary intake and eating behaviors.

Similarly, the Celiac Symptom Index (CSI) was developed to assess symptoms and celiacrelated health at a single point in time. Scores greater than a threshold level of 45 are associated with lower adherence to GFD and worse quality of life.[6] The CSI is based on the manifestations of relevant symptoms while the DIET-GFD monitors diet and behavior related items apart from days of work or school missed due to CD symptoms (which were not reported by most participants). Thus, the correlation between the two measures was extremely low.

A GFD rating system developed by Biagi et al[30] has been validated in an Italian population[27][27][27][27].[27] This five-point scale is determined by an algorithm with items pertaining to voluntary gluten ingestion, disclosure of GFD needs to others, label reading and use of certified gluten-free foods. Unfortunately, the scale is not universally applicable because certified gluten-free foods are not widely available in much of the world, including Canada; therefore, we created a modified Biagi score, which reduced the scale from five to four. This scale has the advantage of being relatively simple to administer and has demonstrated some discriminative ability to predict positive antibodies and abnormal biopsies; $23 \%$ of patients categorized as strictly adherent had positive antibodies and $7 \%$ had abnormal biopsies.[27] In addition, 39\% of patients categorized as non-adherent had normal antibodies and normal biopsies. This lack of specificity limits the use of this scale in the individual patient. In this study, the modified Biagi score is moderately correlated with DIET-GFD. We also noticed that the modified Biagi score classified more participants into the lower half of the scale ( score $=1$ or 2 ) compared to either GF-EAT or DIET-GFD.

Most recently, Wessels et al developed a standardized diet interview consisting of 26 questions with domains including GFD compliance, attitudes towards gluten and patient (parent) knowledge for pediatric patients.[31] This assessment tool was more sensitive than a short questionnaire (modified from the Biagi score) and follow-up antibodies, but the authors did not examine agreement between different dietitians. This scale uses numeric scoring to classify individuals into one of three categories: strict GFD, GFD with important errors, and GFD not followed. The relevance of this scale to adult patients is unknown.

Serum tTG IgA is a sensitive and specific marker of villous atrophy in undiagnosed patients with $\mathrm{CD}$ consuming an unrestricted diet. In the absence of a highly sensitive and specific marker of persistent villous atrophy on a GFD, tTG IgA is the laboratory test most commonly used as a proxy for GFD adherence, both in clinical practice and in research studies. In the present study, serum antibody levels had the lowest correlation with the DIETGFD, or with any other measure of GFD adherence. This result is in keeping with a recent systematic review of the role of antibody testing in predicting mucosal recovery 23 and consistent whether tTG level was considered as a categorical (above/below upper limit of normal) or continuous variable (multiples of the upper limit of normal). Truly sensitive and specific serum markers of CD activity are urgently needed. 
In the absence of such serum markers, diet assessment by a dietitian with expertise in GFDs remains the clinical criterion standard. As the first structured assessment and rating system for dietitians to report GFD adherence, the DIET-GFD has several advantages over existing systems. It is standardized, comprehensive and reproducible. The assessment tool is broad in its scope and includes domains such as grocery shopping and label reading skills, managing gluten in the home and kitchen, eating outside the home, ability to ascertain if food prepared by others is gluten-free, awareness and prevention of cross-contact and intentional dietary lapses. Many of these domains assess risk of gluten ingestion rather that the absolute amount of gluten that may have been ingested. This provides the dietitian with information on high risk behavior to direct counselling to optimize gluten adherence for the individual patient.

There are a few limitations of this study. The dietitians that performed the nutrition assessment and evaluated this tool each had more than ten years of experience in managing patients with CD and thus were very familiar with GFDs and gluten contamination. It would be useful to assess the performance of this scale when utilized by dietitians with less experience in $\mathrm{CD}$, to evaluate its broader utility. Secondly, this study demonstrated good inter-rater agreement, but did not evaluate the correlation with histopathological changes.

\section{Conclusions}

The DIET-GFD is a comprehensive tool to evaluate GFD adherence and risks for gluten ingestion in adults that has good interobserver agreement and may be a useful tool in research protocols and for following individuals on a GFD. Future studies are needed to evaluate whether this tool can be applied reliably by dietitians with less expertise in glutenfree diets and whether the ratings correlate with mucosal recovery.

\section{Acknowledgements}

The authors thank the participants in the Manitoba Celiac Disease Cohort who generously provided their time and shared their experiences.

Funding: This work was supported by the Canadian Institutes of Health Research, the Manitoba Health Research Council (now Research Manitoba), and the Canadian Celiac Association JA Campbell Fund. JAS received salary support from the Canadian Institutes of Health Research, Canadian Association of Gastroenterology, Harvard Medical School Eleanor and Miles Shore Fellowship and the National Institutes of Health (T32 DK 07760 and K23 DK119584).

Conflict of interest disclosures: DRD has received research funding from Biomedal S.L. and has served as a consultant for Takeda Pharmaceuticals and Shire Canada and is a member of the Professional Advisory Council of the Canadian Celiac Association. CNB is supported in part by the Bingham Chair in Gastroenterology. He has served on advisory boards of Abbvie Canada, Ferring Canada, Janssen Canada, Shire Canada, Takeda Canada, and Pfizer Canada and as a consultant to Mylan Pharmaceuticals. He has received educational grants from Shire Canada, Abbvie Canada, Takeda Canada, and Janssen Canada and is on the speaker's bureau for Abbvie Canada, Ferring Canada, Shire Canada and Medtronic Canada. JAS has received research funds from Glutenostics LLC, Biomedal S.L. and Cour Pharmaceuticals and served on an advisory board for Takeda Pharmaceuticals. KG, KG, LAG, LR, DW, KW and JRW have nothing to disclose. The authors declare that they have no conflict of interests.

\section{Glossary}

\section{Conceptualization}

Ideas; formulation or evolution of overarching research goals and aims

\section{Methodology}


Development or design of methodology; creation of models

\section{Software}

Programming, software development; designing computer programs; implementation of the computer code and supporting algorithms; testing of existing code components

\section{Validation}

Verification, whether as a part of the activity or separate, of the overall replication/ reproducibility of results/experiments and other research outputs

\section{Formal Analysis}

Application of statistical, mathematical, computational, or other formal techniques to analyze or synthesize study data

\section{Investigation}

Conducting a research and investigation process, specifically performing the experiments, or data/evidence collection

\section{Resources}

Provision of study materials, reagents, materials, patients, laboratory samples, animals, instrumentation, computing resources, or other analysis tools

\section{Data Curation}

Management activities to annotate (produce metadata), scrub data and maintain research data (including software code, where it is necessary for interpreting the data itself) for initial use and later reuse

\section{Writing - Original Draft}

Preparation, creation and/or presentation of the published work, specifically writing the initial draft (including substantive translation)

\section{Writing - Review \& Editing}

Preparation, creation and/or presentation of the published work by those from the original research group, specifically critical review, commentary or revision - including pre-or postpublication stages

\section{Visualization}

Preparation, creation and/or presentation of the published work, specifically visualization/ data presentation

\section{Supervision}

Oversight and leadership responsibility for the research activity planning and execution, including mentorship external to the core team

\section{Project Administration}

Management and coordination responsibility for the research activity planning and execution

\section{Funding Acquisition}


Acquisition of the financial support for the project leading to this publication

\section{References}

[1]. Pekki H, Kurppa K, Maki M, Huhtala H, Sievanen H, Laurila K, et al. Predictors and Significance of Incomplete Mucosal Recovery in Celiac Disease After 1 Year on a Gluten-Free Diet.Am J Gastroenterol 2015;110:1078-85. 10.1038/ajg.2015.155. [PubMed: 26032154]

[2]. Galli G, Esposito G, Lahner E, Pilozzi E, Corleto VD, Di Giulio E, et al. Histological recovery and gluten-free diet adherence: a prospective 1-year follow-up study of adult patients with coeliac disease. Aliment Pharmacol Ther 2014;40:639-47. 10.1111/apt.12893. [PubMed: 25066096]

[3]. Lebwohl B, Sanders DS, Green PHR. Coeliac disease. Lancet (London, England) 2017;6736:1-12.

[4]. Kelly CP, Bai JC, Liu E, Leffler DA. Advances in Diagnosis and Management of Celiac Disease. Gastroenterology 2015;148:1175-86. 10.1053/j.gastro.2015.01.044. [PubMed: 25662623]

[5]. Lebwohl B, Granath F, Ekbom A, Smedby KE, Murray JA, Neugut AI, et al. Mucosal healing and risk for lymphoproliferative malignancy in celiac disease: a population-based cohort study. Ann Intern Med 2013;159:169-75. 10.7326/0003-4819-1593-201308060-00006. [PubMed: 23922062]

[6]. Leffler DA, Dennis M, Edwards George J, Jamma S, Cook EF, Schuppan D, et al. A validated disease-specific symptom index for adults with celiac disease. Clin Gastroenterol Hepatol 2009;7:1328-34, 1334.e1-3. 10.1016/j.cgh.2009.07.031. [PubMed: 19665584]

[7]. Tau C, Mautalen C, De Rosa S, Roca A, Valenzuela X. Bone mineral density in children with celiac disease. Effect of a Gluten-free diet. Eur J Clin Nutr 2006;60:358-63. [PubMed: 16319837]

[8]. Freeman HJ. Adult celiac disease and its malignant complications. Gut Liver 2009;3:237-46. 10.5009/gnl.2009.3.4.237. [PubMed: 20431755]

[9]. Lee J, Clarke K. Effect of clinical and laboratory parameters on quality of life in celiac patients using celiac disease-specific quality of life scores. Scand J Gastroenterol 2017;52:1235-9. 10.1080/00365521.2017.1350283. [PubMed: 28685635]

[10]. Cossu G, Carta MG, Contu F, Mela Q, Demelia L, Elli L, et al. Coeliac disease and psychiatric comorbidity: epidemiology, pathophysiological mechanisms, quality-of-life, and gluten-free diet effects. Int Rev Psychiatry 2017;29:489-503. 10.1080/09540261.2017.1314952. [PubMed: 28681625]

[11]. White LE, Bannerman E, Gillett PM. Coeliac disease and the gluten-free diet: a review of the burdens; factors associated with adherence and impact on health-related quality of life, with specific focus on adolescence. J Hum Nutr Diet 2016;29:593-606. 10.1111/jhn.12375. [PubMed: 27214084]

[12]. Shah S, Akbari M, Vanga R, Kelly CP, Hansen J, Theethira T, et al. Patient perception of treatment burden is high in celiac disease compared with other common conditions. Am $\mathrm{J}$ Gastroenterol 2014;109:1304-11. 10.1038/ajg.2014.29. [PubMed: 24980880]

[13]. Rubio-Tapia A, Hill ID, Kelly CP, Calderwood AH, Murray JA. ACG clinical guidelines: diagnosis and management of celiac disease. Am J Gastroenterol 2013;108:656-76; quiz 677. [PubMed: 23609613]

[14]. Dewar DH, Donnelly SC, McLaughlin SD, Johnson MW, Ellis HJ, Ciclitira PJ. Celiac disease: management of persistent symptoms in patients on a gluten-free diet. World J Gastroenterol 2012;18:1348-56. 10.3748/wjg.v18.i12.1348. [PubMed: 22493548]

[15]. Leffler DA, Dennis M, Edwards George JB, Jamma S, Magge S, Cook EF, et al. A simple validated gluten-free diet adherence survey for adults with celiac disease. Clin Gastroenterol Hepatol 2009;7:530-6, 536.e1-2. 10.1016/j.cgh.2008.12.032. [PubMed: 19268725]

[16]. Leffler DA, Dennis M, Hyett B, Kelly E, Schuppan D, Kelly CP. Etiologies and predictors of diagnosis in nonresponsive celiac disease. Clin Gastroenterol Hepatol 2007;5:445-50. 10.1016/ j.cgh.2006.12.006. [PubMed: 17382600]

[17]. Abdulkarim AS, Burgart LJ, See J, Murray JA. Etiology of nonresponsive celiac disease: results of a systematic approach. Am J Gastroenterol 2002;97:2016-21. 10.1111/ j.1572-0241.2002.05917.x. [PubMed: 12190170] 
[18]. Högberg L, Grodzinsky E, Stenhammar L. Better Dietary Compliance in Patients with Coeliac Disease Diagnosed in Early Childhood. Scand J Gastroenterol 2003;38:751-4. 10.1080/00365520310003318. [PubMed: 12889562]

[19]. Ciacci C, Cirillo M, Cavallaro R, Mazzacca G. Long-Term Follow-Up of Celiac Adults on Gluten-Free Diet: Prevalence and Correlates of Intestinal Damage. Digestion 2002;66:178-85. 10.1159/000066757. [PubMed: 12481164]

[20]. Moreno M de L, Cebolla Á, Muñoz-Suano A, Carrillo-Carrion C, Comino I, Pizarro Á, et al. Detection of gluten immunogenic peptides in the urine of patients with coeliac disease reveals transgressions in the gluten-free diet and incomplete mucosal healing. Gut 2017;66:250-7. 10.1136/gutjnl-2015-310148. [PubMed: 26608460]

[21]. Comino I, Real A, Vivas S, Síglez MÁ, Caminero A, Nistal E, et al. Monitoring of gluten-free diet compliance in celiac patients by assessment of gliadin 33-mer equivalent epitopes in feces. Am J Clin Nutr 2012;95:670-7. 10.3945/ajcn.111.026708. [PubMed: 22258271]

[22]. Silvester JA, Weiten D, Graff LA, Walker JR, Duerksen DR. Is it gluten-free? Relationship between self-reported gluten-free diet adherence and knowledge of gluten content of foods. Nutrition 2015;32:777-83. 10.1016/j.nut.2016.01.021.

[23]. Silvester JA, Kurada S, Szwajcer A, Kelly CP, Leffler DA, Duerksen DR. Tests for Serum Transglutaminase and Endomysial Antibodies Do Not Detect Most Patients With Celiac Disease and Persistent Villous Atrophy on Gluten-free Diets: a Meta-analysis. Gastroenterology 2017;153:689-701.e1. 10.1053/j.gastro.2017.05.015. [PubMed: 28545781]

[24]. Hall NJ, Rubin G, Charnock A. Systematic review: adherence to a gluten-free diet in adult patients with coeliac disease. Aliment Pharmacol Ther 2009;30:315-30. 10.1111/ j.1365-2036.2009.04053.x. [PubMed: 19485977]

[25]. Simpson S, Thompson T. Nutrition assessment in celiac disease. Gastrointest Endosc Clin N Am 2012;22:797-809. 10.1016/j.giec.2012.07.010. [PubMed: 23083994]

[26]. Silvester JA, Graff LA, Rigaux L, Walker JR, Duerksen DR. Symptomatic suspected gluten exposure is common among patients with coeliac disease on a gluten-free diet. Aliment Pharmacol Ther 2016;44:612-9. 10.1111/apt.13725. [PubMed: 27443825]

[27]. Biagi F, Bianchi PI, Marchese A, Trotta L, Vattiato C, Balduzzi D, et al. A score that verifies adherence to a gluten-free diet: a cross-sectional, multicentre validation in real clinical life. $\mathrm{Br} \mathrm{J}$ Nutr 2012;108:1884-8. 10.1017/S0007114511007367. [PubMed: 22321199]

[28]. Landis JR, Koch GG. The Measurement of Observer Agreement for Categorical Data Published by : International Biometric Society Stable URL : http://www.jstor.org/stable/2529310. Biometrics 1977;33:159-74. 10.2307/2529310. [PubMed: 843571]

[29]. Suchy FJ, Brannon PM, Carpenter TO, Fernandez JR, Gilsanz V, Gould JB, et al. National Institutes of Health Consensus Development Conference Statement on Celiac Disease, June 2830, 2004. Gastroenterology 2005;128:S1-9. 10.1059/0003-4819152-12-201006150-00248. [PubMed: 15825115]

[30]. Biagi F, Andrealli A, Bianchi PI, Marchese A, Klersy C, Corazza GR. A gluten-free diet score to evaluate dietary compliance in patients with coeliac disease. Br J Nutr 2009;102:882-7. 10.1017/ S0007114509301579. [PubMed: 19331704]

[31]. Wessels MMS, te Lintelo M, Vriezinga SL, Putter H, Hopman EG, Mearin ML. Assessment of dietary compliance in celiac children using a standardized dietary interview. Clin Nutr 2017:1-5. 10.1016/j.clnu.2017.04.010. [PubMed: 28935438] 


\section{Highlights}

- The Dietitian Integrated Evaluation Tool for Gluten-free diets (DIET-GFD) is a newly developed 10 point scale that assesses risk and adherence to a glutenfree diet.

- Interobserver agreement between two dietitians using the Dietitian Integrated Evaluation Tool for Gluten-free diets was excellent $(\kappa=0.845)$.

- $\quad$ The 10 point DIET-GFD scale demonstrates that many patients following a gluten-free diet are at risk of gluten exposure but underestimate this risk on self report. 


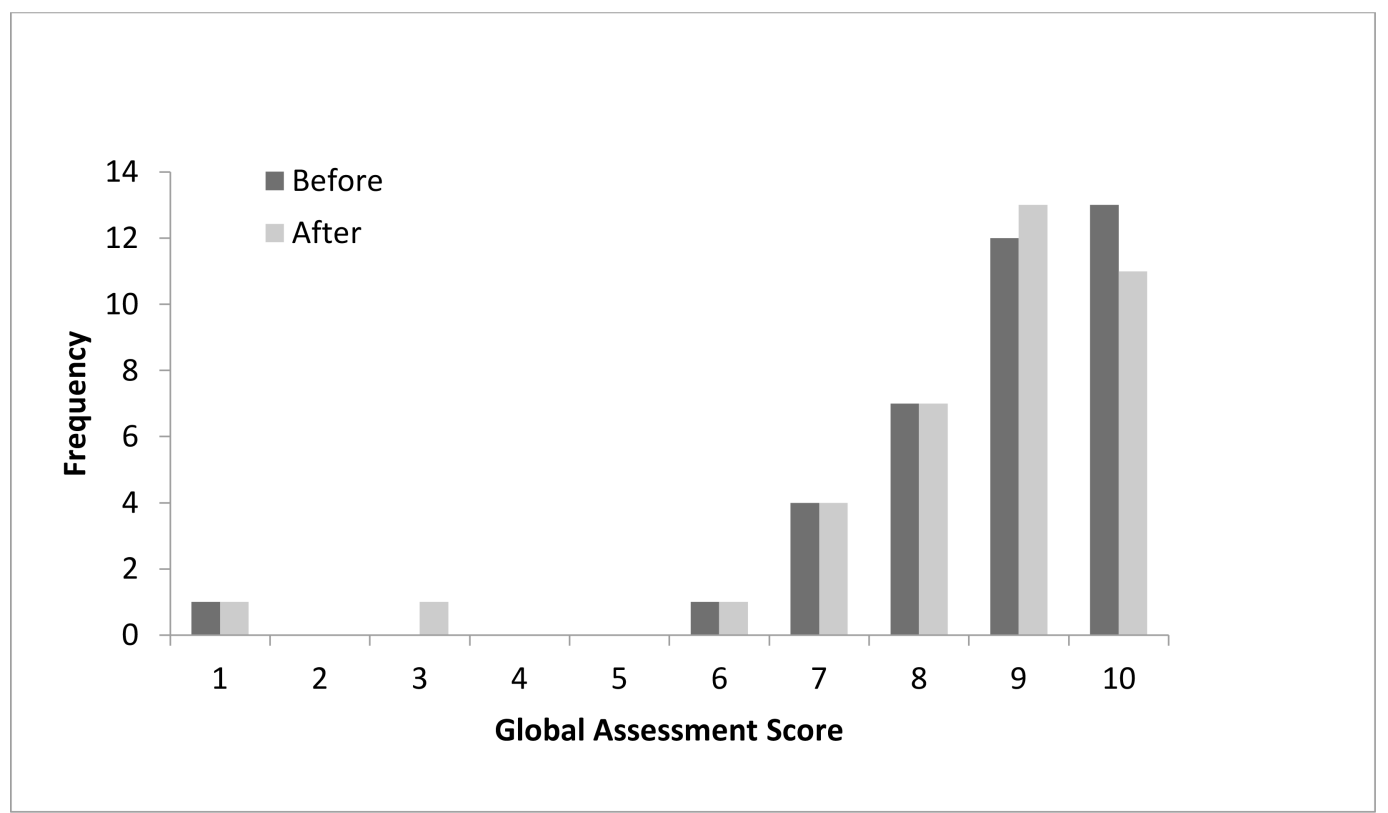

Figure 1.

Self-assessment of gluten-free diet adherence before and after completing report of high-risk behaviors. 




Figure 2.

Comparison of dietitian global assessment of adherence rated by two dietitians. 
Risk at home

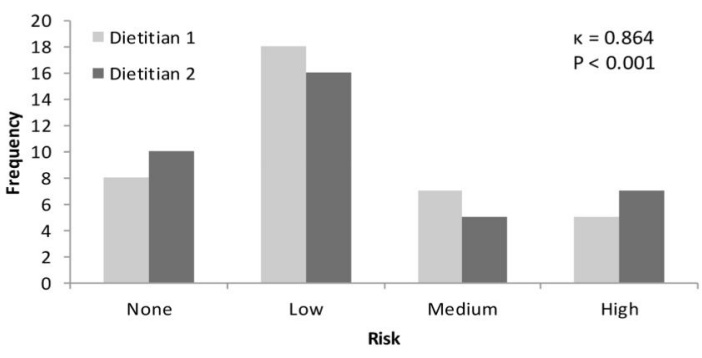

Risk at others' homes

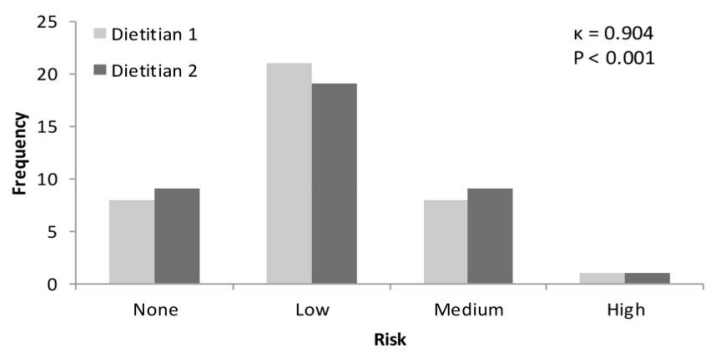

Risk shopping/label reading

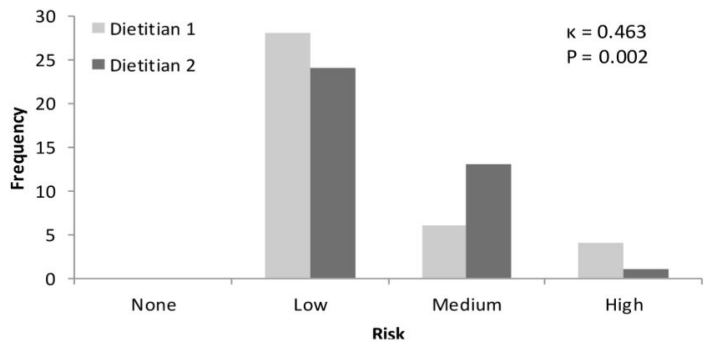

Risk at restaurants

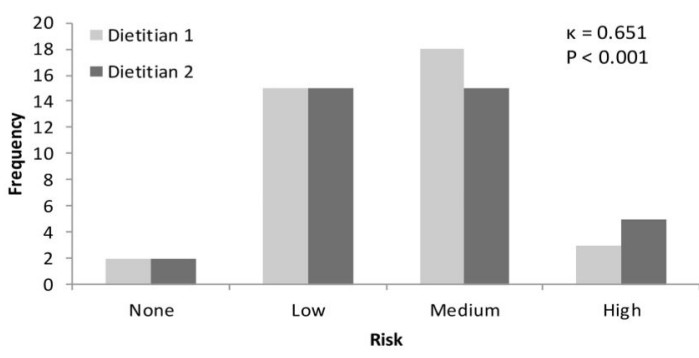

FFQ and food record GFD adherence estimate

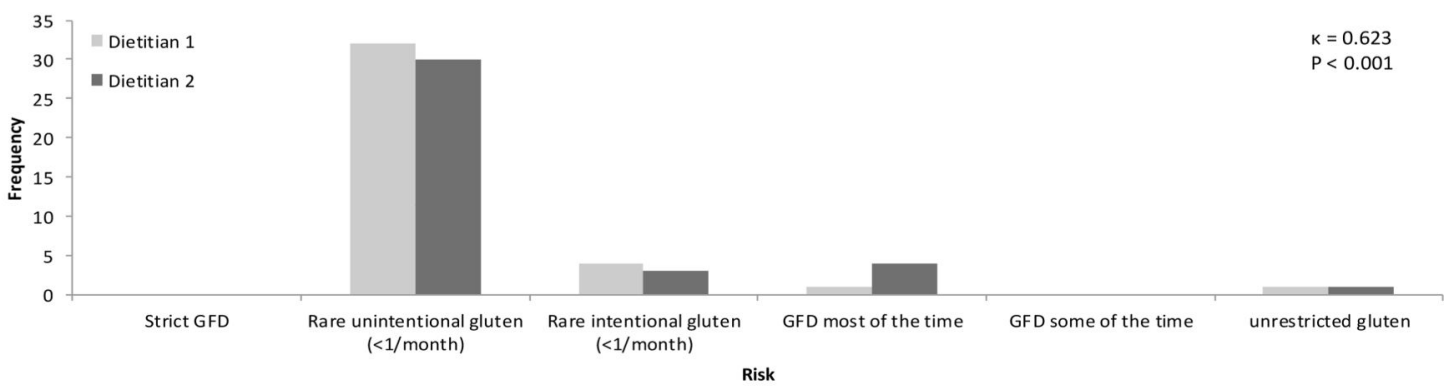

FFQ and food record gluten exposure estimate

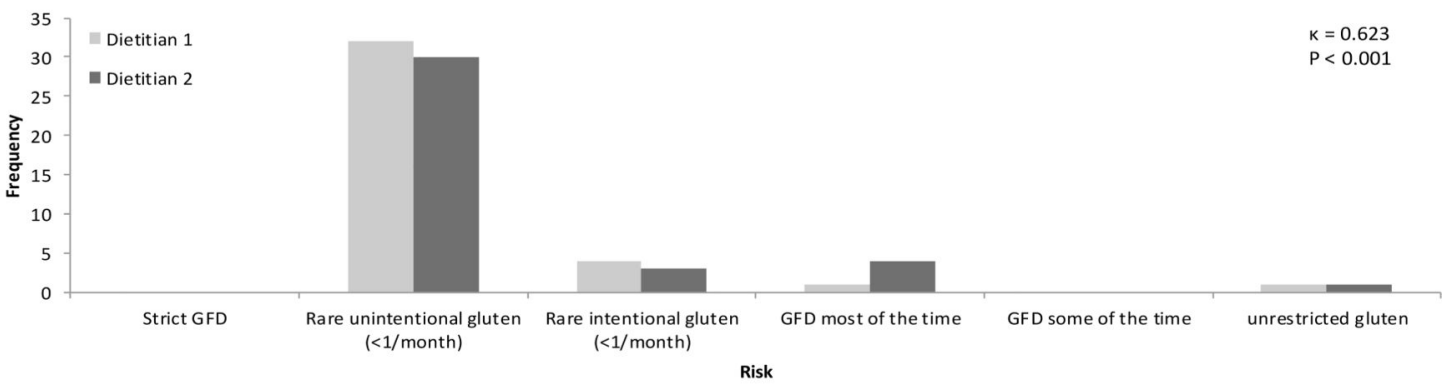

Figure 3.

Dietitian scoring of sub-scales of gluten exposure risk rated by two dietitians. 
Table 1.

The DIET-GFD global assessment score (10-point ordinal descriptive scale)

\begin{tabular}{|c|c|}
\hline Score & Description \\
\hline 1 & $\begin{array}{l}\text { Has gluten in kitchen; No precautions with storage, condiments, food prep; Uses bulk bins/deli; }>5 \text { foods on food frequency that } \\
\text { contain gluten; Eats out }>1 \text { week and does not ask appropriate gluten precaution questions; consumes gluten when eating out; Eats } \\
\text { foods that contain gluten as a major ingredient }>1 / \text { week. }\end{array}$ \\
\hline 2 & $\begin{array}{l}\text { Has gluten in kitchen; No precautions with storage, condiments, food prep; Uses bulk bins/deli; Has }>10 \text { foods on frequency that } \\
\text { likely contain gluten or }>5 \text { foods that contain gluten; Eats out }>1 \text { time per month and does not ask appropriate gluten precaution } \\
\text { questions; Eats foods that contain gluten as a major ingredient } 1-4 \text { times/month. }\end{array}$ \\
\hline 3 & $\begin{array}{l}\text { Has gluten in kitchen; No separate condiment/margarine or storage of GF items; Uses bulk bins/deli; Has }>7 \text { foods on frequency that } \\
\text { likely contain gluten or }>3 \text { foods that contain gluten; Eats out at least once per month and does not ask appropriate gluten precaution } \\
\text { questions; Eats foods that contain gluten as a major ingredient }<\times 1 / \text { month. }\end{array}$ \\
\hline 4 & $\begin{array}{l}\text { Has gluten in kitchen; No separate condiments/margarine; no separate storage of GF items; Uses bulk bins/deli; Has }>5 \text { foods on } \\
\text { frequency that likely contain gluten or }>1 \text { food that contains gluten; Eats out }>1 / \text { week; answers SOMETIMES or RARELY for } \\
\text { questions about restaurant gluten precautions; Eats foods that contain gluten as a moderate ingredient regularly or major ingredient on } \\
\text { occasion. }\end{array}$ \\
\hline 5 & $\begin{array}{l}\text { Has gluten in kitchen; No separate condiment/margarine; Uses bulk bins/deli; Has }>5 \text { foods on frequency that likely contain gluten; } \\
\text { Eats out > 1/week; answers SOMETIMES for questions about restaurant gluten precautions; Eats food that he/she "thinks" is GF or } \\
\text { "may" be GF; Eats gluten as a moderate ingredient on occasion or as a minor ingredient regularly. }\end{array}$ \\
\hline 6 & $\begin{array}{l}\text { Has gluten in kitchen; Has > } 3 \text { foods on frequency that likely contain gluten; Eats out > 1/week; orders food he/she "thinks" is GF; } \\
\text { Eats food that "may" be GF. }\end{array}$ \\
\hline 7 & $\begin{array}{l}\text { Has gluten in kitchen OR no gluten in kitchen, but not as careful with eating out; No separate condiments/margarine; Uses bulk bins/ } \\
\text { deli; Has } 3+\text { foods on frequency that likely contain gluten; Eats out }>\text { once per month; answers SOMETIMES for questions about } \\
\text { restaurant gluten precaution eating. }\end{array}$ \\
\hline 8 & $\begin{array}{l}\text { Has gluten in kitchen but takes all precautions or does not use condiments/margarine; Uses bulk bins/deli; }<3 \text { foods on frequency that } \\
\text { likely contain gluten; Eats out up to } 1 / \text { month; asks MOST questions about restaurant gluten precaution eating. }\end{array}$ \\
\hline 9 & $\begin{array}{l}\text { Has gluten in kitchen, but takes all precautions or does not use condiments/margarine; No bulk bins, no deli; Eats out up to once per } \\
\text { month; asks ALL restaurant questions. }\end{array}$ \\
\hline 10 & No gluten in kitchen; Rarely eats out (e.g. few times/year) and asks ALL questions about restaurant gluten precaution eating \\
\hline
\end{tabular}

$\mathrm{GF}=$ Gluten-free 
Table 2.

Participant characteristics $(\mathrm{n}=37)$

\begin{tabular}{|c|c|}
\hline Characteristic & \\
\hline Age [median $(\mathrm{Q} 1, \mathrm{Q} 3)]$ & $40(32,57)$ \\
\hline Female $[N(\%)]$ & $26(62 \%)$ \\
\hline \multicolumn{2}{|l|}{ HLA Genotype [N (\%)] } \\
\hline DQ2 only & $32(87 \%)$ \\
\hline DQ8 only & $3(8 \%)$ \\
\hline DQ2 and DQ8 & $2(5 \%)$ \\
\hline tTG IgA (multiples of upper limit of normal) & $0.87(0.41,1.57)$ \\
\hline \multicolumn{2}{|l|}{ Gluten-Free Eating Assessment Tool (GF-EAT) [N (\%)] } \\
\hline Frequent gluten (more than once per week) & $1(3 \%)$ \\
\hline Occasional gluten (1-4 times per month) & $1(3 \%)$ \\
\hline Rare intentional gluten exposure ( $<1 /$ month $)$ & $3(8 \%)$ \\
\hline Rare accidental gluten exposure (<1/month) & $29(76 \%)$ \\
\hline No gluten & $4(10 \%)$ \\
\hline Celiac Symptom Index (CSI) [median(Q1, Q3)] & $30(25,36)$ \\
\hline Above symptom threshold $(>45)^{+}[\mathrm{N}(\%)]$ & $2(5 \%)$ \\
\hline Celiac Disease Adherence Test (CDAT) [median(Q1, Q3)] & $10(9,12)$ \\
\hline Inadequate adherence $(>13)[\mathrm{N}(\%)]$ & $3(8 \%)$ \\
\hline \multicolumn{2}{|l|}{ Modified Biagi score $[\mathrm{N}(\%)]$} \\
\hline 0 & $5(13 \%)$ \\
\hline 1 & $1(3 \%)$ \\
\hline 2 & $14(37 \%)$ \\
\hline 3 & $18(47 \%)$ \\
\hline
\end{tabular}

${ }^{+}$CSI scores > 45 are associated with both lower adherence and quality of life. 
Table 3.

Dietitian assessment of adherence

\begin{tabular}{|c|c|c|}
\hline & Dietitian 1 & Dietitian 2 \\
\hline Dietitian global assessment of adherence & $8(7,8)$ & $7(7,8)$ \\
\hline \multicolumn{3}{|l|}{ Risk at home } \\
\hline None & $10(26 \%)$ & $8(21 \%)$ \\
\hline Low & $16(42 \%)$ & $18(47 \%)$ \\
\hline Medium & $5(13 \%)$ & $7(18 \%)$ \\
\hline High & $7(18 \%)$ & $5(13 \%)$ \\
\hline \multicolumn{3}{|l|}{ Risk at others' homes } \\
\hline None & $9(24 \%)$ & $8(21 \%)$ \\
\hline Low & $19(50 \%)$ & $21(55 \%)$ \\
\hline Medium & $9(24 \%)$ & $8(21 \%)$ \\
\hline High & $1(3 \%)$ & $1(3 \%)$ \\
\hline \multicolumn{3}{|l|}{ Risk shopping/label reading } \\
\hline None & $0(0 \%)$ & $0(0 \%)$ \\
\hline Low & $24(63 \%)$ & $28(74 \%)$ \\
\hline Medium & $13(34 \%)$ & $6(16 \%)$ \\
\hline High & $1(3 \%)$ & $4(11 \%)$ \\
\hline \multicolumn{3}{|l|}{ Risk at restaurants } \\
\hline None & $2(5 \%)$ & $2(5 \%)$ \\
\hline Low & $15(41 \%)$ & $15(41 \%)$ \\
\hline Medium & $15(41 \%)$ & $17(46 \%)$ \\
\hline High & $5(14 \%)$ & $3(8 \%)$ \\
\hline \multicolumn{3}{|c|}{ FFQ and food record GFD adherence estimate } \\
\hline Strict GFD & $0(0 \%)$ & $0(0 \%)$ \\
\hline Unintentional gluten $<1 /$ month & $30(79 \%)$ & $32(84 \%)$ \\
\hline Intentional gluten $<1$ /month & $3(8 \%)$ & $4(11 \%)$ \\
\hline GFD most of the time & $4(11 \%)$ & $1(3 \%)$ \\
\hline GFD some of the time & $0(0 \%)$ & $0(0 \%)$ \\
\hline Unrestricted gluten & $1(3 \%)$ & $1(3 \%)$ \\
\hline \multicolumn{3}{|c|}{ FFQ and food record gluten exposure estimate } \\
\hline No gluten & $0(0 \%)$ & $0(0 \%)$ \\
\hline Crumbs $(<50 \mathrm{mg})$ & $32(84 \%)$ & $32(84 \%)$ \\
\hline$<1 / 4$ slice $(<0.5 \mathrm{~g})$ & $4(11 \%)$ & $2(5 \%)$ \\
\hline$<1 / 2$ slice $(<1 \mathrm{~g})$ & $1(3 \%)$ & $0(0 \%)$ \\
\hline$<1$ slice $(<2 \mathrm{~g})$ & $0(0 \%)$ & $2(5 \%)$ \\
\hline$\geq 10$ slice ( $\geq 20 \mathrm{~g}$ ) & $0(0 \%)$ & $1(3 \%)$ \\
\hline
\end{tabular}

FFQ = Food Frequency Questionnaire 
Table 4.

Correlation between dietitian global assessment of adherence and other assessment methods

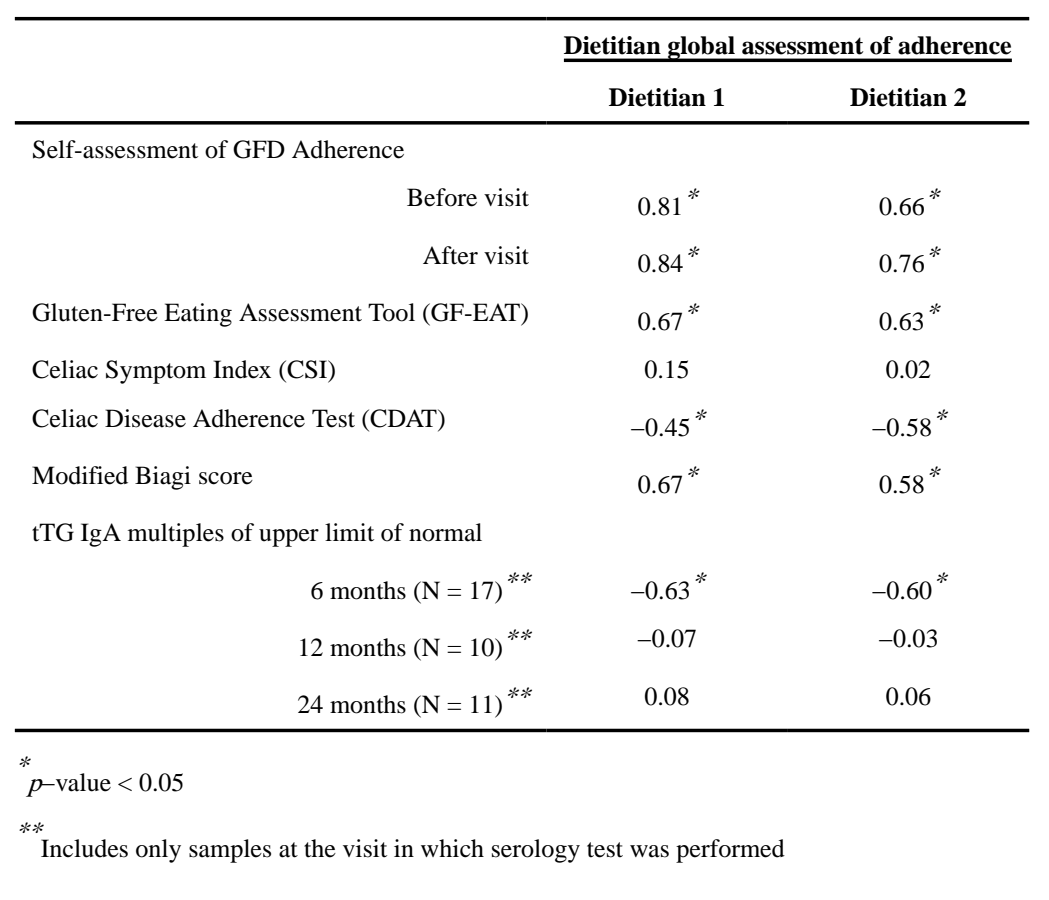

